\title{
A Measurement-based Power System Model for Dynamic Response Estimation and Instability Warning
}

\author{
Yong Liu*, Kai Sun, Yilu Liu \\ *Corresponding author: yliu66@utk.edu \\ Department of Electrical Engineering, University of Tennessee, Knoxville, TN, 37996, U.S.
}

\begin{abstract}
Traditional power system dynamic models are very complex due to the intricacy of power system networks and can be inaccurate for control because of the amount of details needed and the fact that the grid topology changes all the time. With the fast deployment of a large number of phasor measurement units (PMUs) in the transmission network, a purely measurement-based model for dynamics estimation and control may be obtained. After a preliminary examination of the strong linearity of large-scale power systems, this paper proposes a linear autoregressive with exogenous input model (ARX)-structured power system dynamic model and demonstrates that this proposed model is effective in power system dynamic response estimation and instability warning. Both simulation and real measurement data have been used to validate the proposed method.
\end{abstract}

Index Terms-Autoregressive with exogenous input (ARX) model, dynamic response estimation, power system dynamic model, phasor measurement unit (PMU), instability warning.

\section{INTRODUCTION}

The dynamic model of a power system plays a vital role in power system planning and operations. An accurate dynamic model should be able to faithfully reveal system responses to different disturbances and help establish an accurate stability boundary so that a grid can be operated at its full capacity while staying within the stability region [1] [2]. Therefore, the identification and validation of power system dynamic models has always been a focus of researchers and engineers [3]-[7]. However, traditional power system simulation models are very complex due to the intricacies of power system networks. Those models can also be inaccurate because of multiple layers of equipment and their operational intricacies involved in the modern bulk grid, and due to the fact that power system topologies constantly change as breakers open or close, lines are taken out of service and generators are re-dispatched. In the present power grid operating paradigm, detailed dynamic models are usually updated once every few months or less often, and thus, it's not surprising that discrepancies in simulation models would limit the performance of online applications for power system stability and control.

On the other hand, even for a high-dimensional complex power system, only a limited number of transfer functions of the system are critical to determine its stability and control actions, which means a reduced power system model may be developed and identified online for stability analysis and control purposes[1]. Especially with the availability of a large number of synchrophasors being deployed [8]-[10], it is possible to dynamically construct that reduced power system model based purely on real-time measurements to reflect most recent changes in the system.

In this paper, based on a preliminary analysis of large-scale power systems' linearity, a linear power system dynamic model in the autoregressive with exogenous input (ARX) model structure is proposed. The proposed model is based purely on widearea time-domain measurement data and identified in near real-time by a least squares technique to reflect the minor changes 
that occur in the bulk power system. Unlike traditional linear system models that are derived from nonlinear equations around an operating point with a series of simplifications and constraints [1] [2], the proposed model will not encounter the computational burden and modeling errors that linearization creates, especially when considering large-scale power systems. Since it can be updated on the order of seconds using a new set of measurement data, the proposed model is expected to have potential benefits for dynamics estimation and control.

This paper is structured as follows. In Section II, the large-scale power system's linearity is discussed; in Section III, a brief description of the linear ARX model structure and model accuracy index is given; in Section IV, a concept of power system dynamic response estimation is introduced, an example is used to describe its basic procedure, and then case studies using both simulation and measurement data are given to substantiate the proposed method's effectiveness; in Section V, a potential application of the proposed method in power system instability warning is introduced using case studies. In the last section, the whole paper is summarized.

\section{DISCUSSION OF THE LINEARITY OF LARGE-SCALE POWER GRIDS}

From the point of view of control theory, a power grid is naturally a nonlinear network. That is why almost all the power system modeling and simulation tools currently available are designed to handle a large number of differential algebraic equations. Accepting the nonlinearity of certain components, power system small-signal studies, which are considered as an important part of the general problem of power system stability, state that the linearization around certain operation points may be achieved if no obvious or large disturbances occurs [11][12]. However, the strong linearity in large-scale power systems may has been grossly underestimated. In this section, the existence of a large-scale power system's strong linearity will be preliminarily discussed.

Superposition principles should apply for any system considered as linear. In this section, a superposition method is applied to test a large-scale power system's linearity. The U.S. Eastern Interconnection (EI) model is taken as an example here (basic information of this model is shown in Table I). First, two disturbances of similar magnitude at different locations are simulated individually using the 16,000-bus EI model. Both disturbances are $800 \mathrm{MW}$-level generation losses (the most typical disturbance in reality). System responses (including frequency, voltage magnitude and angle) are recorded at a fixed monitoring point following these two disturbances. Then, another simulation that includes both disturbances is carried out. According to the superposition principles, if the EI system is linear, the sum of two individual disturbances' responses should be identical to the result of the third simulation that includes both disturbances. Fig. 1 illustrates one case study using this test methodology and shows how the superposition principle is met nearly perfectly for frequency and voltage angle. Apparently, the linearity of the EI system is almost perfect in this case. In fact, this test has been carried out repeatedly and the EI system has always demonstrated very linear characteristics unless the disturbances were extremely large (e.g., over 4,000 MW). The study illustrates how the conventional definition of "small-signal" and understanding of power system "linearity" has been too conservative; hence, this valuable property of the grid has been underutilized in many critical ways.

\section{Proposed Linear Power System Dynamic Model Structure}

Considering a large-scale power system's strong linearity, a linear power system dynamic model structure may be accurate enough for power system dynamics study and control. Here, an ARX model structure is used, which will be briefly introduced below. 


\section{A. ARX Model Structure}

As a common linear model structure, the ARX model provides a much simpler model identification problem of a multivariable system than state-space and other models [13]. The mathematical structure expression of the ARX model is given below:

$A_{k}(a) \hat{n}_{k}(t)=\sum_{j=1}^{n_{j}} B_{j k}(z) u_{j}(t)+e(t)$

where $t$ is the sampled data number, $\boxminus(t)$ is the system noise, $u_{j}$ and $\hat{y}_{k}$ is the model's $j$-th input and $k$-th output, respectively. $A_{i k}(z)$ and $B_{j k}(z)$ are the ARX nominator and denominator polynomials respectively, which are given by:

$A_{h 2}(z)=1+a_{h i} z^{-1}+\cdots+a_{h m_{n_{k}}} z^{-n n_{k}}$

$B_{j h}(z)=b_{j h o}+b_{j h 1} z^{-1}+\cdots+b_{j h\left(m_{j k}-1\right)} z^{-\left(m_{j k}-1\right)}$

where $n_{a_{k}}$ and $n_{b_{j k}}$ are the orders of the model, and $j$ and $k$ are the numbers of inputs and outputs, respectively. The model parameters of a multi-variable ARX model can be estimated by a linear least squares technique. The least squares estimation problem is solved by using QR factorization to optimize the ARX model parameters. The details of ARX structure and algorithm are discussed in [13]. After obtaining the model orders and parameters, the identified models can be evaluated by comparing their outputs with the actual system outputs.

\section{B. Model Accuracy Index}

As discussed above, a common ARX model structure may be employed in the measurement-based model identification process to describe power system dynamics. In order to evaluate the identified models' quality, a model accuracy index defined below can be calculated for each output over a time window (assuming that the window has $L$ data points):

Accurac $y_{k}=\left\{1-\frac{\sqrt{\sum_{l=1}^{2}\left[y_{k}\left[D-y_{k}[]^{2}\right.\right.}}{\sqrt{\sum_{=1}^{2} W_{k}\left[D-\gamma_{k}^{2}\right.}}\right\} \times 100$

where $y_{k}(b)$ and $\hat{y}_{k}(b)$ are the measured and estimated responses of the $k$-th output at the $l$-th data point respectively, and $\bar{y}_{k}$ is the mean value of measured response over the time window. This index is the most widely used evaluation index in system identification. It is mainly used to reflect the accuracy of the model in describing dynamic characteristics and thus suitable to describe the accuracy of the ARX model here. An index of 100 means a perfect match between the estimated response and measured response, and the model identified can fully reflect the dynamic characteristics of the system. If the index is 0 , it means the estimated response is no better than the mean value of measured response. In that case, the identified model cannot describe the system's dynamic characteristics. It should be noted that, this index can also be negative, which means the estimated response and measured response are not related at all.

\section{DyNAMIC RESPONSE ESTIMATION}

\section{A. Concept of Dynamic Response Estimation}

Energy Management System (EMS) plays a very important role in the monitoring and control of power systems, and state estimation forms its backbone. Because of the extensive installations of PMUs in the power system, traditional power system state estimation has been undergoing an essential change. Basically, the literature [14]-[17] brought forward some methods that combined the mixed measurements from PMUs and SCADA in state estimation after PMUs were placed at some buses and 
improved the accuracy of traditional state estimation. However, power system state estimation, in its current status, is not capable of capturing the system dynamic behavior (except for some very slow system changes). It only provides monitoring information in the form of a sequence of steady states, or quasi-steady states (uniquely defined as a set of voltage magnitudes and phase angles at different buses in the network). This in turn limits the wide area control actions of the system to very slow steady state control that is usually executed by the system operators manually. In addition, automatic control during dynamic situations is provided only very locally in the current control paradigm, not taking into consideration the wide-area system dynamic behavior which can be tracked precisely by PMUs. Dynamic State Estimation (DSE) techniques with their unique ability to predict the state vector one time stamp ahead have the potential to foresee potential contingencies and security risks [18] [19]. However, dynamic state estimation still fails to estimate more transient or dynamic states of a power system. Therefore, any improvement in the ability to predict or estimate a power system's dynamic characteristics will definitely go a long way in reducing the security risks in the modern power system.

To achieve the goal of dynamics prediction or estimation in a power system, a concept of dynamic response estimation is proposed in this paper. Unlike traditional state estimation and dynamic state estimation techniques that focus on estimating relatively stationary state vectors such as bus voltage magnitudes during steady and quasi-steady states, dynamic response estimation seeks to estimate or predict the dynamic response of a power system during transient situations. The basic idea of dynamic response estimation is to identify the real-time dynamic model or transfer function of the power system and use the obtained model to estimate or predict the power system's dynamic response. ARX model structure and least squares techniques will be employed to identify the necessary dynamic model for response estimation. Due to the precise dynamic measurement capabilities of PMUs, the model identification will be based purely on PMU measurements. A procedure of dynamic response estimation is given in the following subsection.

\section{B. Procedure of Dynamic Response Estimation}

Detailed procedure of the proposed dynamic response estimation method is illustrated by a specific example in this part. Assuming there are five high voltage buses located in the U.S. Tennessee area with PMU measurements, which are shown in Fig. 2, the basic steps of dynamic response estimation is 1) to identify the power system dynamic model between the "output bus" (green ones in Fig. 2) and the "input buses" (yellow ones in Fig.2) using PMU measurement data of certain contingencies, and 2) to use the trained power system dynamic model and the measurement data from the "input buses" to estimate the dynamic response of the "output bus" of other contingencies. The dynamic contingencies (or events) which data are used to train the model are referred to as the "excitation sources" of the model, whose locations are also indicated in Fig.2.

In the later part of this section, case studies are employed to demonstrate the effectiveness of the proposed dynamic response estimation method. In subsection C, "artificial" PMU measurement generated by PSS $^{\circledR}$ E dynamic simulation using the U.S. Eastern Interconnection model are utilized in the case study while in subsection D real synchrophasor measurement data are applied.

\section{Case Studies using Simulated Data}

A series of dynamic contingencies, including generation trip, load shedding, line trip and line fault that occur in the U.S. Eastern Interconnection are simulated in $\mathrm{PSS}^{\circledR} \mathrm{E}$ using the 16,000-bus EI dynamic model. Simulation data of the five buses in Fig.2 from these dynamic contingencies, including frequency, voltage magnitude and voltage angle, are treated as "artificial" PMU measurements to train the proposed dynamic response estimation model. As mentioned above, these dynamic contingencies (or events) are referred to as excitation sources of the model. The response estimated from the model and the actual response from $\mathrm{PSS}^{\circledR} \mathrm{E}$ will be compared to evaluate the accuracy of the trained model. 
Theoretically, all the disturbances in power system, including generation trip, load shedding, line trip, and fault, should be able to serve as excitation sources of the proposed model. Because a number of relatively large events happen every day in the U.S. power system (as shown in Fig. 3), it is very likely there will be sufficient excitations for the model's construction, which lays the foundation for the proposed dynamic response estimation's field applications.

However, while the dynamic system response of generation trip and load shedding cases can be monitored in a wide-area sense, line trip events can only be seen in a relatively small part of the grid. Therefore, it is likely that the models excited by different disturbances will have different levels of system coverage extent and accuracy. Therefore, considering the possible influence of different types of excitation sources on the accuracy of the dynamic response estimation model, a comprehensive set of cases studies has been completed to evaluate the proposed method and check whether all the events are acceptable excitation sources. The excitation source events and estimated events are all shown in Table II. Four different types of events (of similar size) that occur in the New York area are used as excitation source events to train four sets of models. Then, the simulation data of a generation trip in Florida (the estimated event) are applied to the four models to perform the dynamic response estimation respectively. Frequency, voltage, and angle response estimation are given in Fig. 4, 5 and 6, respectively.

From the comparisons between the actual and model-estimated dynamic responses in Fig. 4, 5 and 6, it is obvious that the model-estimated responses fit the actual ones well in all cases. This means when the PMU measurement data from certain important bus is missing or incorrect, the proposed dynamic response estimation method can contribute to the estimation or correction of response measurement of that bus. It should be noted that all the types of events occur in the grid may be considered as "excitation sources", though it seems that generation trip and load shedding are better candidates for excitation sources than line trips and line faults as shown by the accuracy index in Fig. 4-6 and Table III. These case studies demonstrate that the measurement-based dynamic model estimation method is promising in the power system dynamics estimation. Though dynamic response estimation still remains in the conceptual stage and may include much broader and deeper extent than what this paper proposes, the preliminary results given in this paper may indicate great improvements in the ability to predict and/or estimate a power system's dynamic characteristics, and thus would go a long way in enhancing the dynamic operation of modern power system.

\section{Case Studies using Real Synchrophasor Data}

Historical event data from the Frequency Monitoring Network (FNET) operated at the University of Tennessee is also used to validate the approach. The FNET system is based on the Frequency Disturbance Recorder (FDR), i.e. a single-phase synchrophasor measuring frequency, voltage magnitude and angle with very high precision at the $120 \mathrm{~V}$ distribution level [20][21]. As shown in Fig. 7, five FDRs deployed in U.S. Midwest area are selected to test the approach. Four FDRs are considered as inputs and one FDR as the output. Their specific locations are marked as red spots on the map in Fig. 7.

Similar to the previous simulation study, one generation trip event (as shown in Fig. 8) detected by FNET system on UTC 21:42:37, Jan. 1, 2012 is used to train the model, and then a load shedding event that happened about 23 minutes later (also indicated in Fig. 8) is used to test the accuracy of the identified model. Because the accuracies of frequency and angle measurements of FDRs have been widely validated, frequency and angle are considered as the model's outputs in this test. The comparisons between estimated frequency/angle response and the real measurement data (FDR 755) are given in Fig. 9 and 10. Apparently, both the estimated frequency and angle responses match the real measurements well.

\section{POTENTIAL APPLICATIONS IN INSTABILITY WARNING}

The application of ARX-structured modeling method is under the assumption that the power system to be modeled is relatively linear around certain operation points. However, when a series of disturbances are increasingly stressing a power grid, 
stability margin of the system will be decreased and its linearity will be destroyed. In this circumstance, the dynamic response estimation result based on the linear models tends to be less accurate and the model accuracy index becomes smaller and smaller as a result, which makes it an effective instability indicator. In this section, a case study will be used to demonstrate the effectiveness of this accuracy index in power system instability warning problem.

Rotor angle instability is one major power system stability issue [1] [2]. Potential of the proposed ARX model in predicting rotor angle instability under cascading outages is introduced in this section and the basic procedure for testing the proposed method is given below:

- First, an angle instability contingency following a sequence of disturbances is designed and simulated on the EI test system using $\mathrm{PSS}^{\circledR} \mathrm{E}$.

- Then, a model in the proposed ARX model structure is identified using a period of system response data following the first disturbance.

- After obtaining that model, system responses following the rest of the disturbance sequence are applied to the model to perform the response estimations. Then the trend of accuracy index will be plotted and examined.

Because the power grid of Florida area is relatively independent of the other part of the EI system, it can be considered as a different control area with the rest of EI system (as shown in Fig.11). Therefore, a sequence of disturbances, including both generation and line trips, are simulated around the interface between these two control areas without causing out of step until the last disturbance (in the rectangle in Fig.11).

The system responses of input and output buses after the line trips or generation trips are shown in Fig.12. It's clear that the Florida-area power grid is synchronized with the other part of EI system until the 5-th trip, after which the Florida area power grid is out of step. The five second system response (frequency) following the first disturbance is used to obtain the model and then the frequency responses following 2-nd to 5-th trips are applied to the model to perform the response estimations.

The accuracy indices of response estimations are shown in Fig.13. From Fig.13, it's obvious that the estimated accuracy decreases gradually with the series of disturbances. After the 4-th disturbance, the accuracy index becomes negative, which implies the Florida area is going to lose synchronism with the rest of the EI system.

\section{DISCUSSION AND CONCLUSION}

This paper proposed a measurement-based power system dynamic modeling method for system response estimation and instability warning, which is based on a reduced dynamic power grid model in the autoregressive with exogenous input model structure. For a large-scale power grid, the identification of the proposed model is computationally efficient and suitable for online applications. Case studies have been conducted to test the proposed model's accuracy and reliability with different excitation sources for model training and cascading events leading to out of step. The preliminary results have shown that the proposed method seems to be effective in estimating power system dynamic response from limited synchrophasor measurements and promising in predicting instability for out of step or other instability issues caused by cascading outages.

As showed by the linearity test in Section II, the linearity of large-scale power systems can be strong. However, it is important to note that the linearity of a power grid could vary significantly from one operation point to another. Therefore, the applications of system linearity should be carefully selected. Furthermore, there does not exist a direct method to measure the linearity of power systems so far. Considering the fact that increasing renewable generations may gradually decrease the overall system linearity, it is necessary to develop a power system linearity measurement method in the future. Furthermore, future work will investigate how linear the power system need to be in order to guarantee the linear modeling accuracy for certain 
power system applications. Although further investigation continues, it seems that based on the proposed method, online synchrophasor-based power system monitoring and decision support applications may be developed for power system response estimation under disturbances and for early warning of potential stability issues.

\section{ACKNOWLEDGEMENT}

This work was supported by Electric Power Research Institute and also made use of Engineering Research Center Shared Facilities supported by the Engineering Research Center Program of the National Science Foundation and DOE under NSF Award Number EEC-1041877 and the CURENT Industry Partnership Program.

\section{BIOGRAPHY}

Yong Liu received his Ph. D. degree in electrical engineering (power system direction) from the University of Tennessee, Knoxville, in 2013. He received his M.S. and B. S. degree in electrical engineering from Shandong University, China, in 2007 and 2010, respectively. He is currently a research assistant professor in the DOE/NSF-cofunded engineering research center CURENT and Department of Electrical Engineering and Computer Science at the University of Tennessee, Knoxville. His research interests are wide-area power system measurement and power system dynamic analysis.

Kai Sun received the B.S. degree in automation in 1999 and the Ph.D. degree in control science and engineering in 2004 both from Tsinghua University, Beijing, China. He was a Postdoctoral Research Associate at Arizona State University, Tempe, from 2005 to 2007, and was a Project Manager in grid operations and planning areas at EPRI, Palo Alto, CA from 2007 to 2012. $\mathrm{He}$ is currently an Assistant Professor at the Department of Electrical Engineering and Computer Science, University of Tennessee, Knoxville, TN, USA. He serves as an editor in IEEE Transactions on Smart Grid.

Yilu Liu received her M.S. and Ph.D. degrees from the Ohio State University, Columbus, in 1986 and 1989. She received the B.S. degree from Xian Jiaotong University, China. Dr. Liu is currently the Governor's Chair at the University of Tennessee, Knoxville and Oak Ridge National Laboratory (ORNL). She is also the deputy Director of the DOE/NSF-cofunded engineering research center CURENT. Prior to joining UTK/ORNL, she was a Professor at Virginia Tech. She led the effort to create the North American power grid Frequency Monitoring Network (FNET) at Virginia Tech, which is now operated at UTK and ORNL as GridEye. Her current research interests include power system wide-area monitoring and control, large interconnection-level dynamic simulations, electromagnetic transient analysis, and power transformer modeling and diagnosis.

\section{REFERENCES}

[1] P. Kundur, Power System Stability and Control, New York: McGraw-Hill, Inc., 1994.

[2] J. Machowski, J. Bialek, and J. Bumby, Power System Dynamics: Stability and Control, New Jersey: John Wiley \& Sons, 2008.

[3] J.P. Barret, P. Bornard, and B. Meyer, Power System Simulation, Berlin: Springer, 1997.

[4] D. N. Kosterev, C. W. Taylor, and W. A. Mittelstadt, "Model Validation for the August 10, 1996 WSCC System Outage," IEEE Trans. on Power Systems, vol. 14, no. 3, pp. 967-979, August 1999.

[5] Z. Huang, P. Du, D Kosterev, and B. Yang, "Application of Extended Kalman Filter Techniques for Dynamic Model Parameter Calibration, " in Proc. IEEE Power and Energy Society General Meeting, 2009, pp: 1-8.

[6] Z. Ning, L. Shuai, R. Singh, and M.A Elizondo, "Calibration of Reduced Dynamic Models of Power Systems using Phasor Measurement Unit (PMU) Data," in Proc. North American Power Symposium (NAPS), 2011, pp: 1-7. 
[7] E. Allen, D.N. Kosterev, P. Pourbeik, "Validation of Power System Models," in Proc. IEEE Power and Energy Society General Meeting, 2010, pp.1-7.

[8] J. S. Thorp and A. G. Phadke, Wide Area Monitoring, Protection and Control, Berlin: Springer, 2012.

[9] A.G. Phadke, J.S. Thorp, and M.G. Adamiak, "A New Measurement Technique for Tracking Voltage Phasors, Local System Frequency, and Rate of Change of Frequency," IEEE Trans. on Power Apparatus and Systems, vol.PAS-102, no.5, pp.1025-1038, May 1983.

[10] J. De La Ree, V. Centeno, J.S. Thorp, and A.G. Phadke, "Synchronized Phasor Measurement Applications in Power Systems," IEEE Trans. On Smart Grid, vol.1, no.1, pp.20-27, June 2010.

[11] G. Rogers, Power System Oscillations. Berlin: Springer, 2000.

[12] A. R. Messina, Inter-area Oscillations in Power Systems: A Nonlinear and Nonstationary Perspective. Berlin: Springer, 2009.

[13] Lennart Ljung, System Identification: Theory for the User (2nd Edition), New Jersey: PTR Prentice, 1999.

[14] A.G. Phadke, J.S. Thorp, K.J. Karimi, "State Estimlatjon with Phasor Measurements," IEEE Trans. on Power Systems, vol.1, no.1, pp.233-238, Feb. 1986.

[15] R. Zivanovic, C. Cairns, "Implementation of PMU technology in State Estimation: an Overview," in Proc. IEEE africon 4th africon conference in africa, 1996, pp: 1006-1011.

[16] J.S. Thorp, A.G. Phadke, K.J. Karimi, "Real Time Voltage-Phasor Measurement For Static State Estimation," IEEE Trans. on Power Apparatus and Systems, vol.PAS-104, no.11, pp.3098-3106, Nov. 1985.

[17] M.J. Rice, G.T. Heydt, "Power Systems State Estimation Accuracy Enhancement Through the Use of PMU Measurements," in Proc. IEEE Transmission and Distribution Conference and Exhibition, 2005/2006, pp:161-165.

[18] G. Durgaprasad and S.S. Thakur, "Robust Dynamic State Estimation of Power Systems based on M-Estimation and Realistic Modeling of System Dynamics,” IEEE Trans. in Power Systems, Vol. 13 , pp. 1331-1336, 1998.

[19] E. Ghahremani, I. Kamwa, "Dynamic State Estimation in Power System by Applying the Extended Kalman Filter With Unknown Inputs to Phasor Measurements," IEEE Trans. on Power Systems,, Vol. 26, Iss. 4, pp. 2556-2566, Nov. 2001.

[20] Z. Zhong, C. Xu, B. J. Billian, L. Zhang, S. S. Tsai, R. W. Conners, V. A. Centeno, A. G. Phadke, and Y. Liu, "Power system Frequency Monitoring Network (FNET) Implementation,” IEEE Trans. on Power Systems, vol. 20, no. 4, pp. 19141921, Nov. 2005.

[21] Y. Zhang, P. N. Markham, T. Xia, L. Chen, Y. Ye, Z. Wu, Z. Yuan, L. Wang, J. Bank, J. Burgett, R. W. Conners, and Y. Liu, "Wide-area Frequency Monitoring Network (FNET) architecture and applications," IEEE Trans. On Smart Grid, vol. 1, no. 2, pp. 159-167, Sept. 2010. 
Fig. 1. U.S. EI system linearity test results

Fig. 2. Illustration of dynamic response estimation

Fig. 3. Disturbances within the U.S. EI system in a single day

Fig. 4. Frequency response estimation

Fig. 5. Voltage response estimation

Fig. 6. Angle response estimation

Fig. 7. FNET deployment of Michigan area

Fig. 8. Generation trip events used for model training

Fig. 9. Comparison of estimated frequency response and actual frequency measurement

Fig. 10. Comparison of estimated angle response and actual angle measurement

Fig. 11. Map of Florida area power grid

Fig. 12. Frequency response following the disturbances

Fig. 13. Accuracy indices of frequency response estimation following the sequence of disturbances 


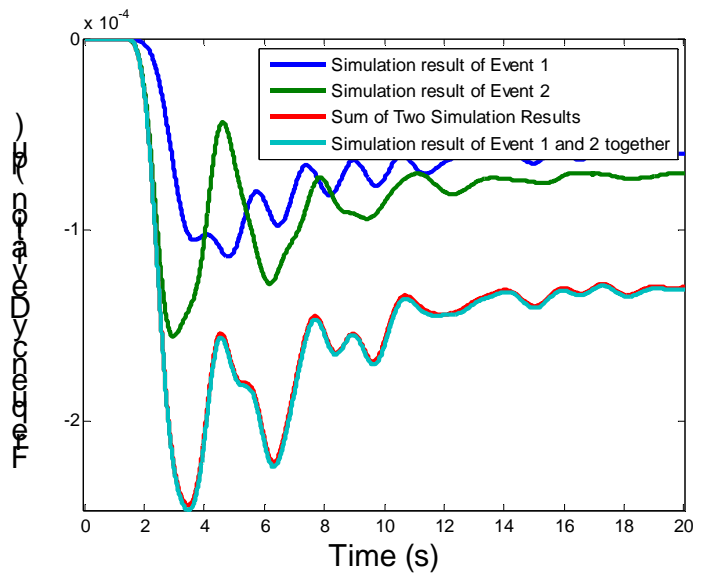

(a) Frequency

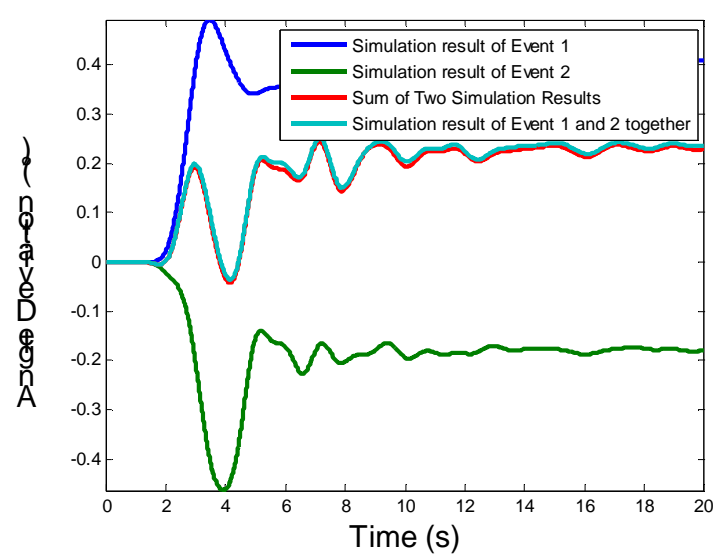

(b) Voltage angle

Fig.1. U.S. EI system linearity test results 


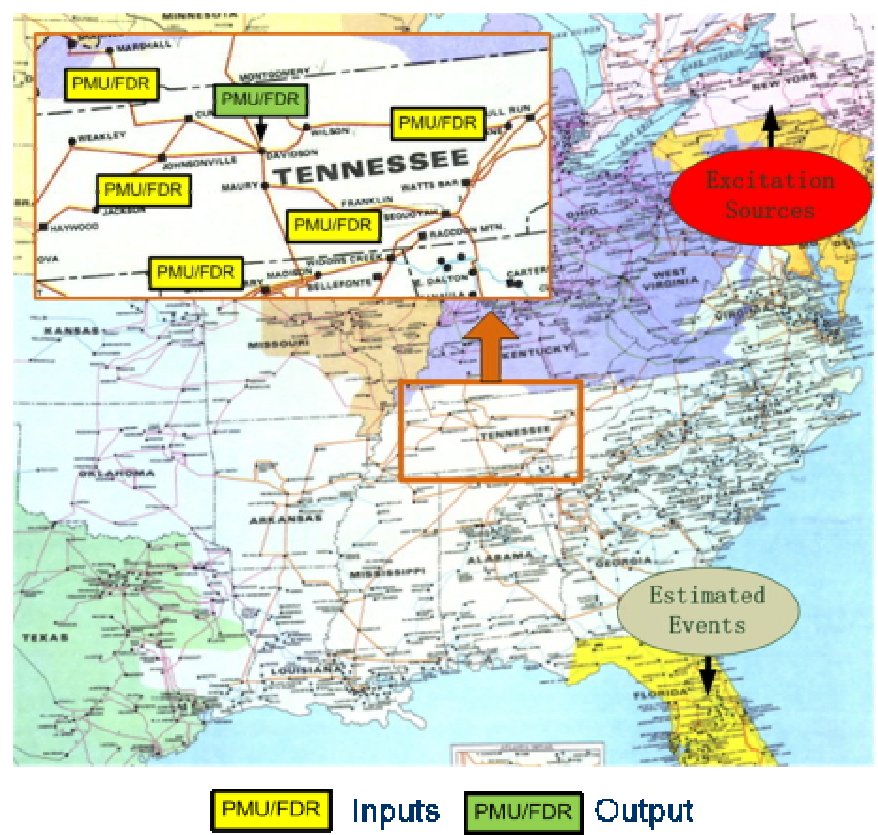

Fig. 2. Illustration of dynamic response estimation

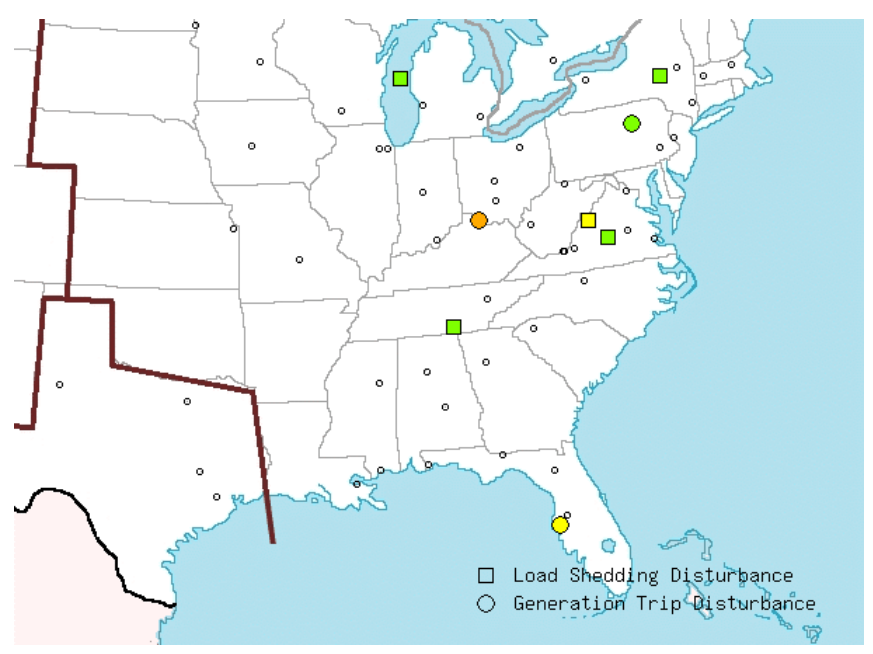

Fig. 3. Disturbances within the U.S. EI system in a single day 


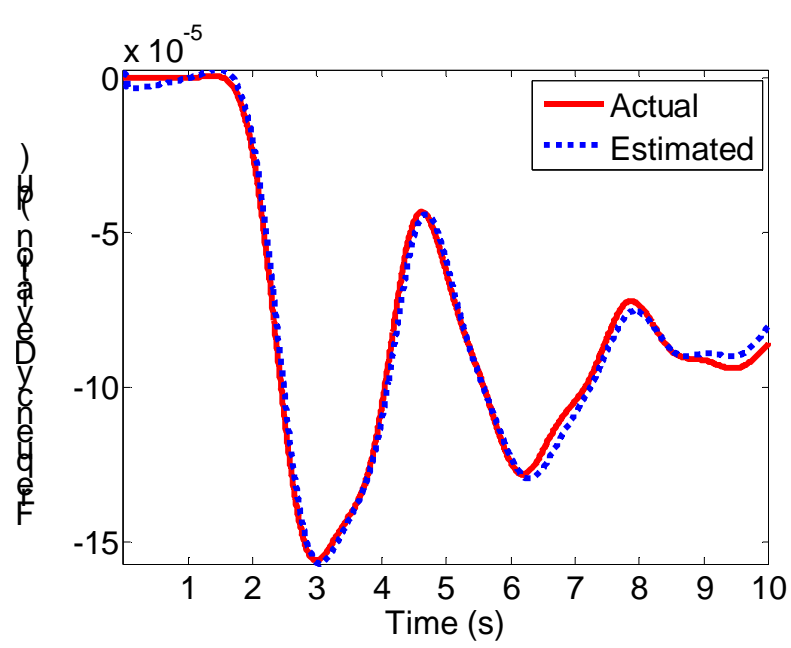

(a) Generation trip as excitation

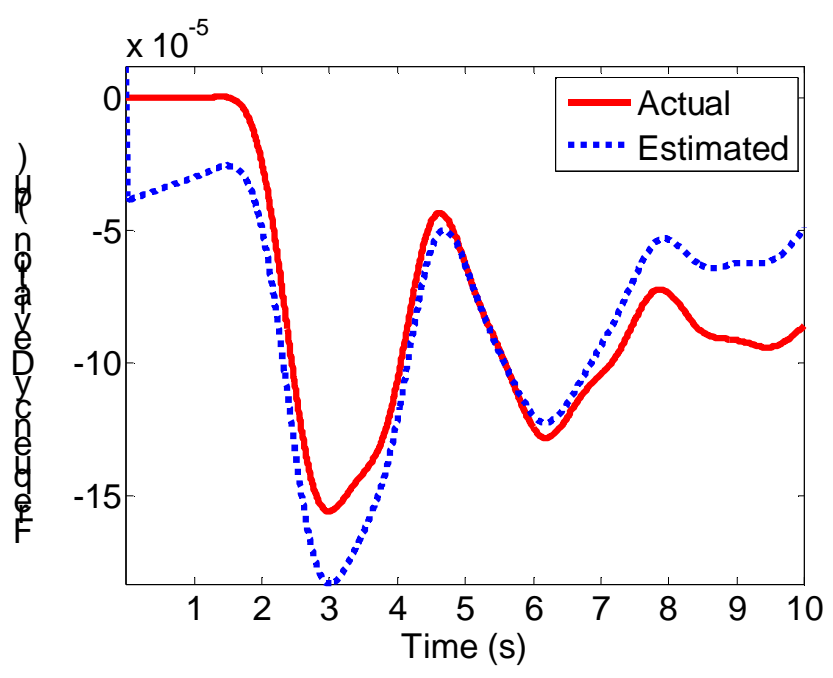

(c) Line trip as excitation

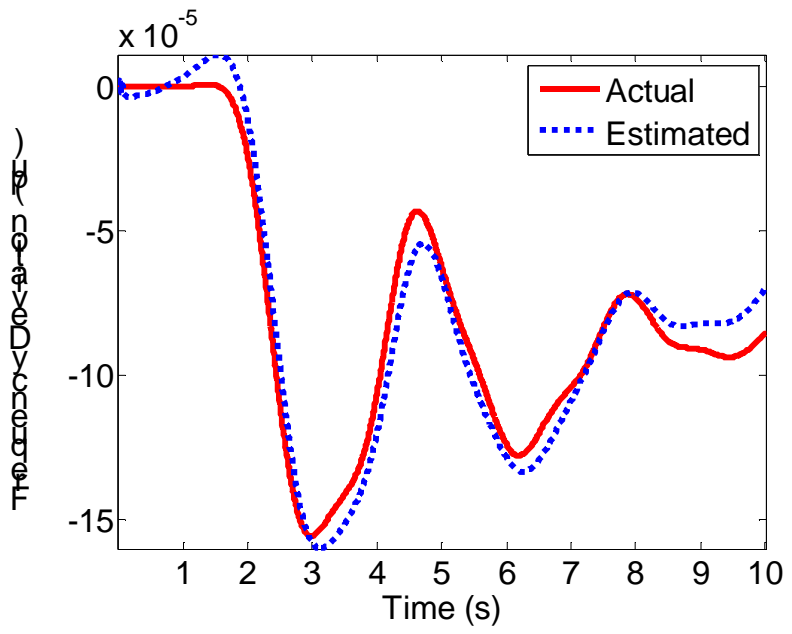

(b) Load shedding as excitation

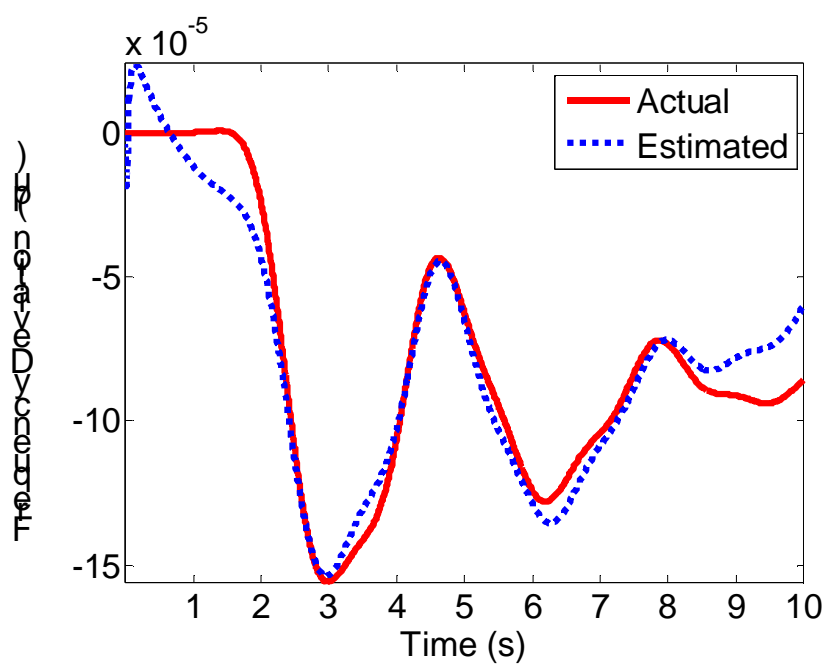

(d) Line fault as excitation

Fig. 4. Frequency response estimation 


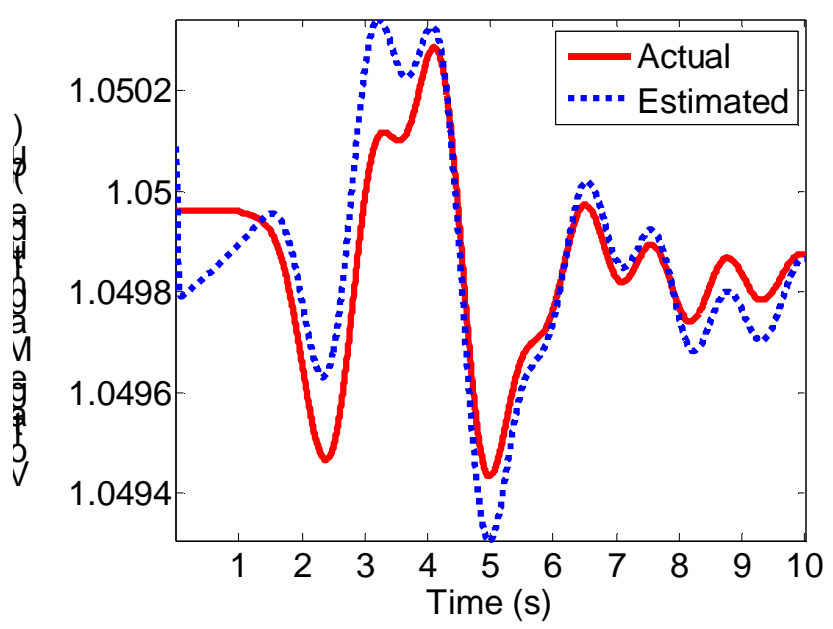

(a) Generation trip as excitation

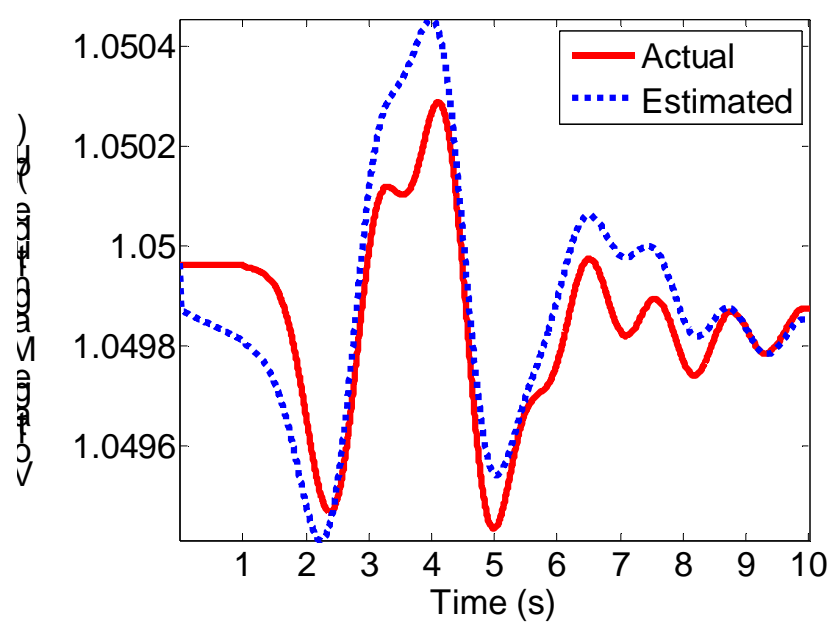

(c) Line trip as excitation

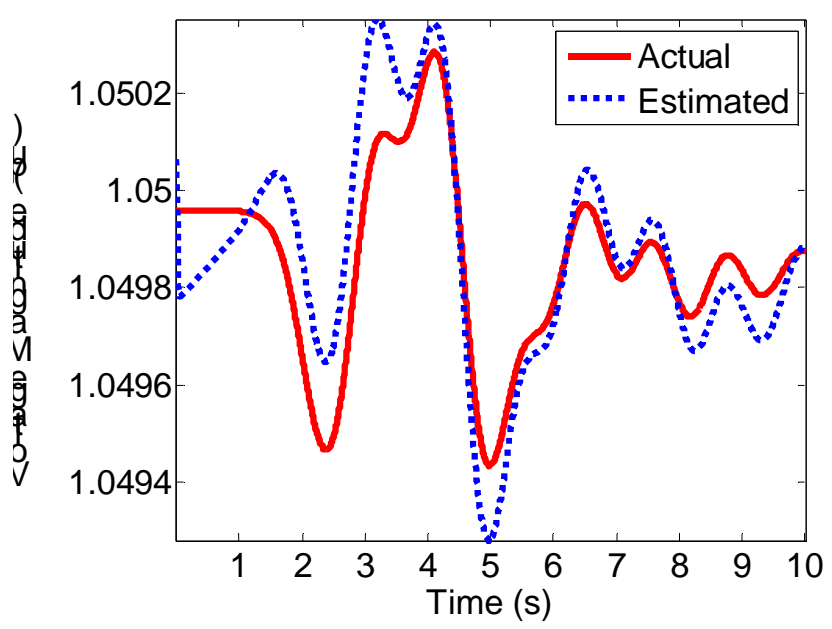

(b) Load shedding as excitation

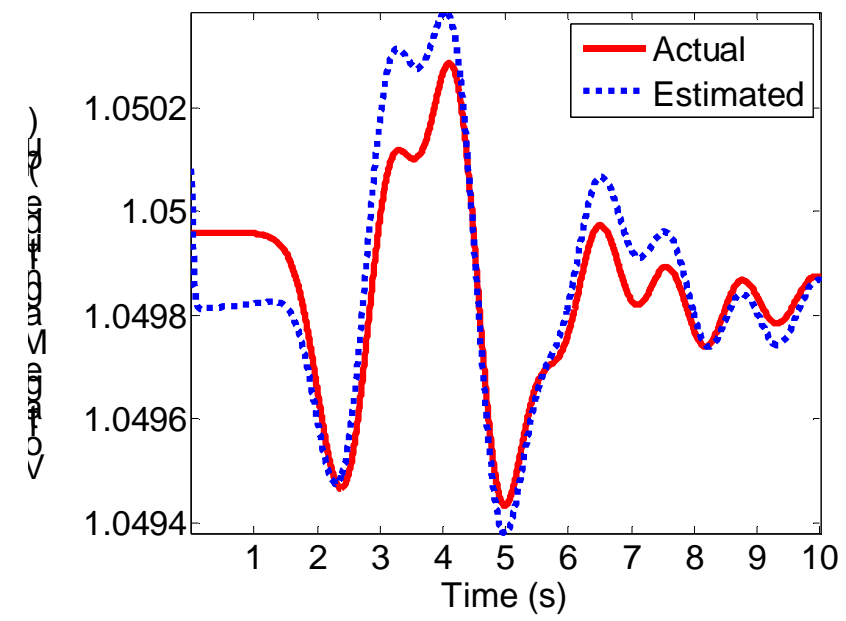

(d) Line fault as excitation

Fig. 5. Voltage response estimation 


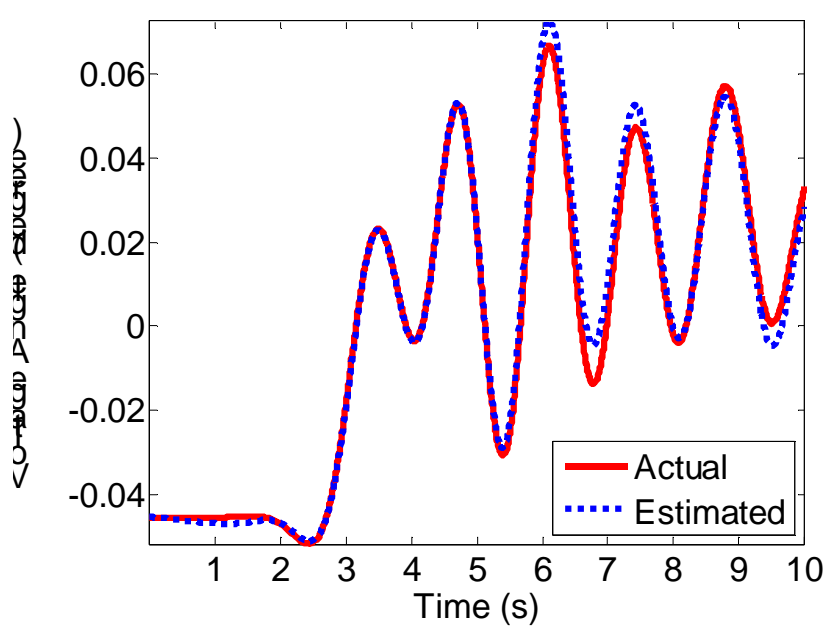

(a) Generation trip as excitation

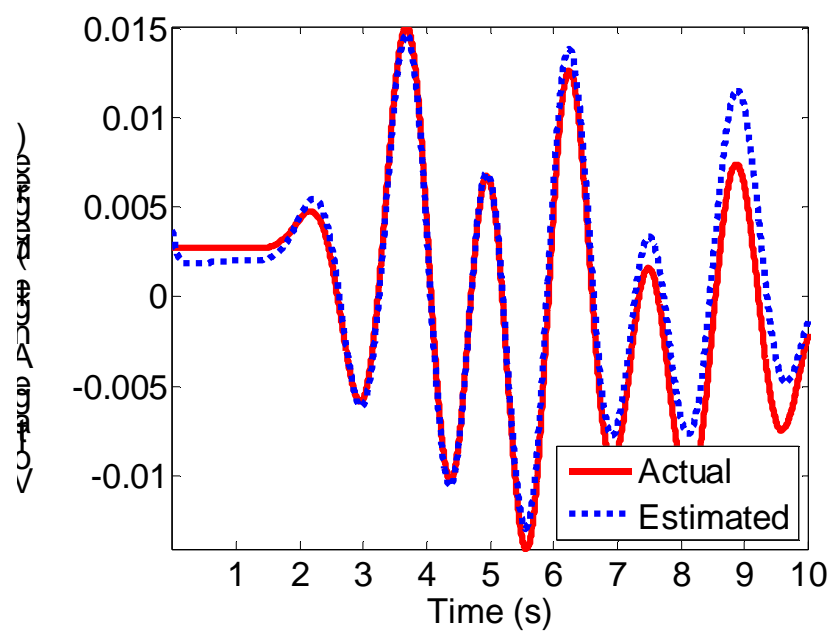

(c) Line trip as excitation

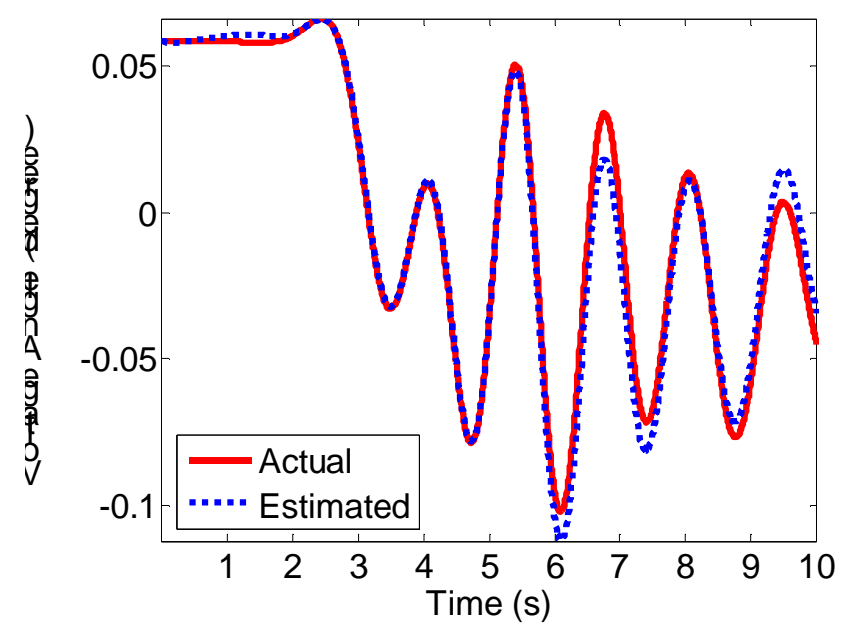

(b) Load shedding as excitation

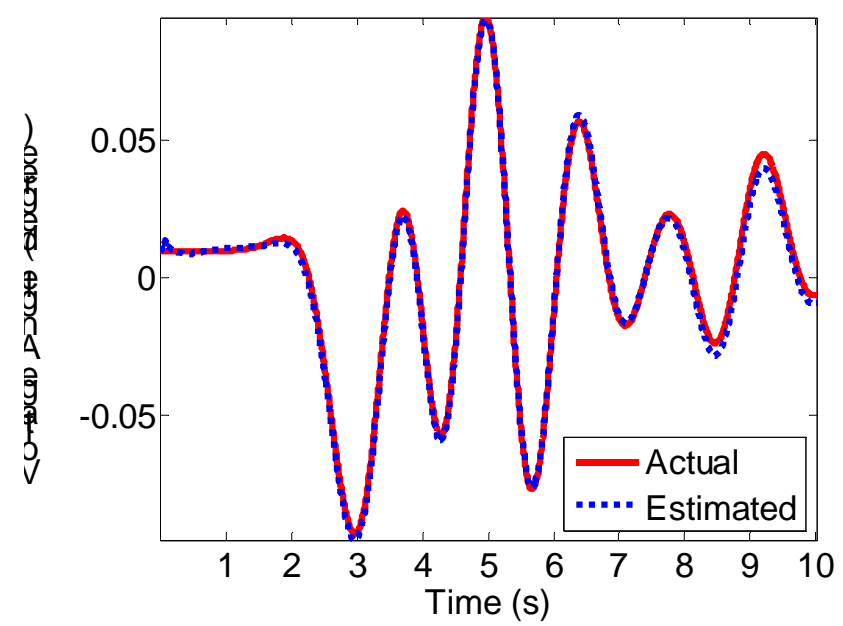

(d) Line fault as excitation

Fig. 6. Angle response estimation 


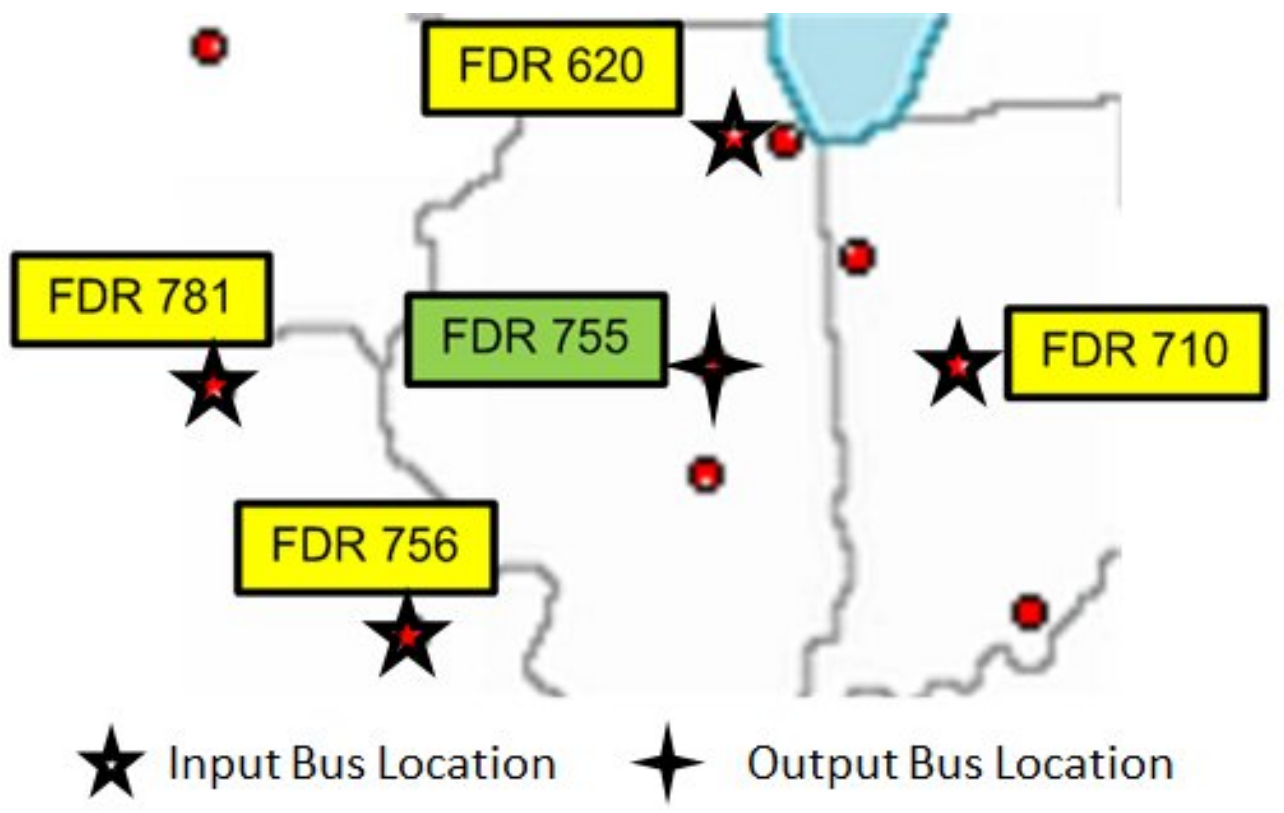

Fig. 7. FNET deployment of Michigan area.

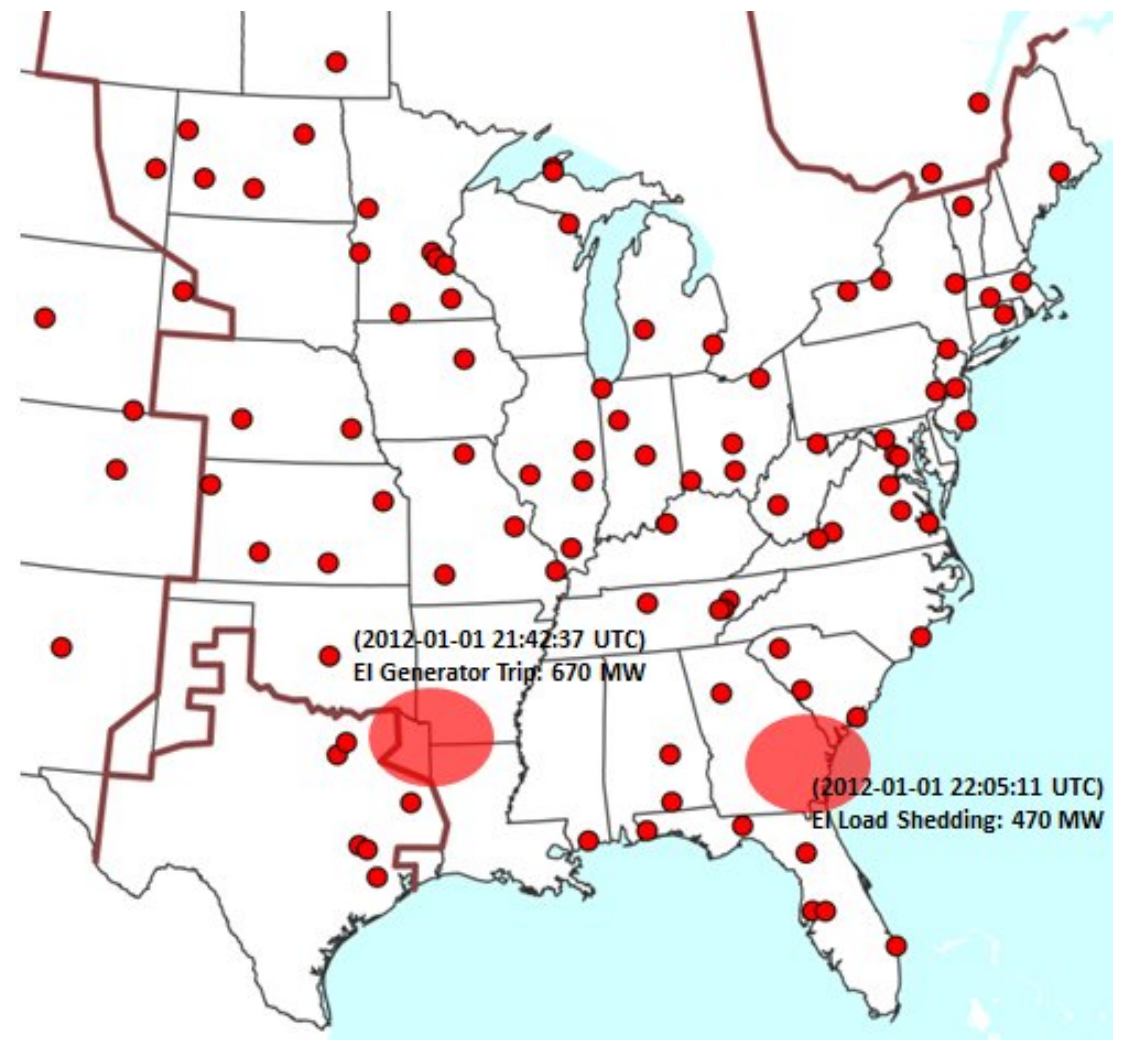

Fig. 8. Generation trip events used for model training. 


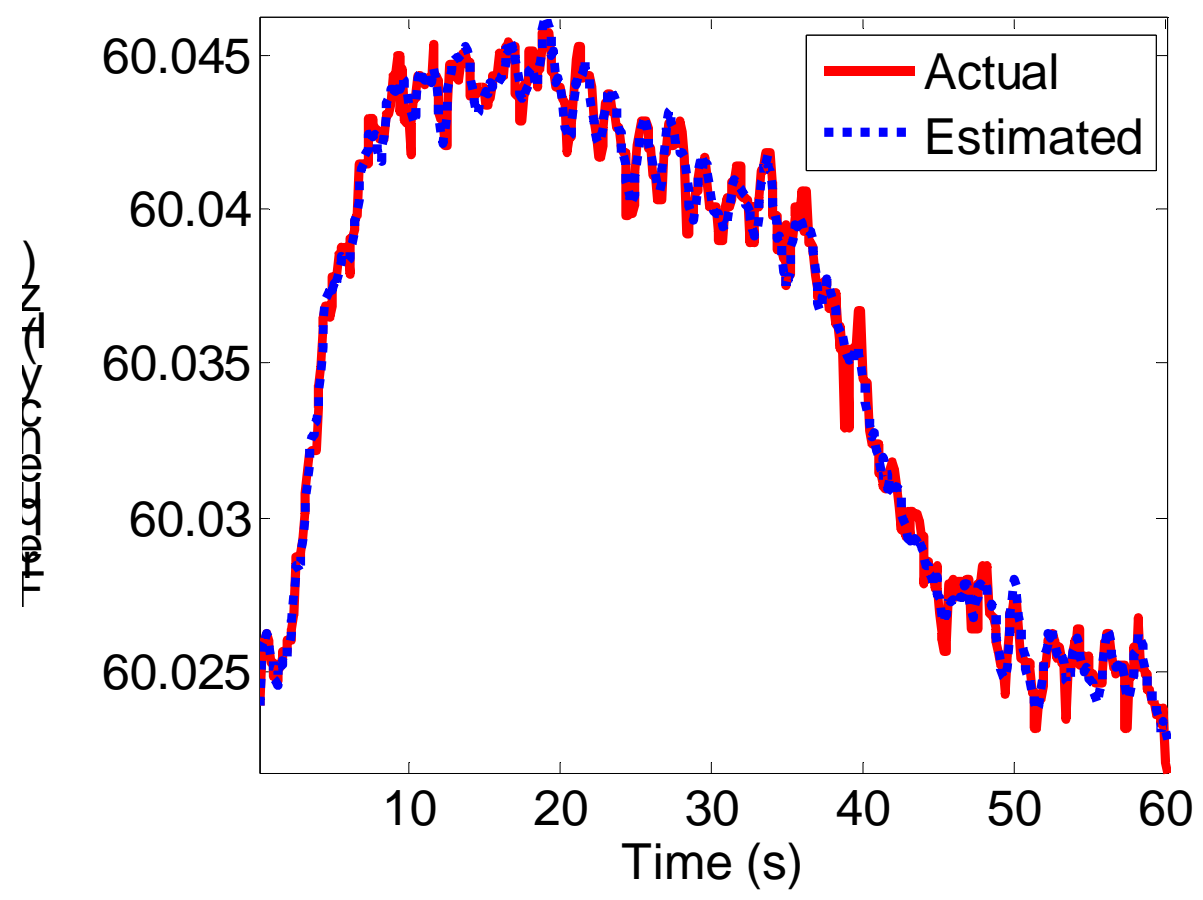

Fig. 9. Comparison of estimated frequency response and actual frequency measurement

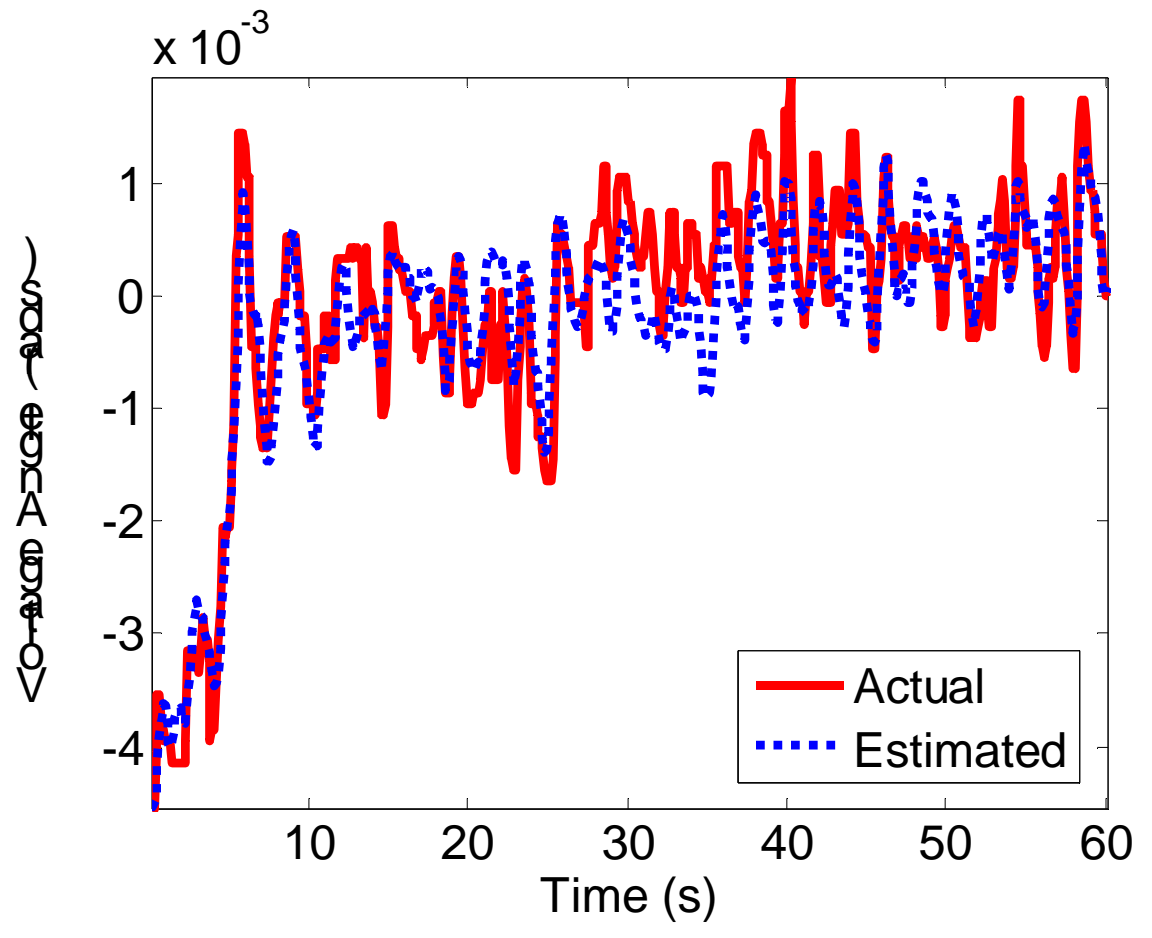

Fig. 10. Comparison of estimated angle response and actual angle measurement 


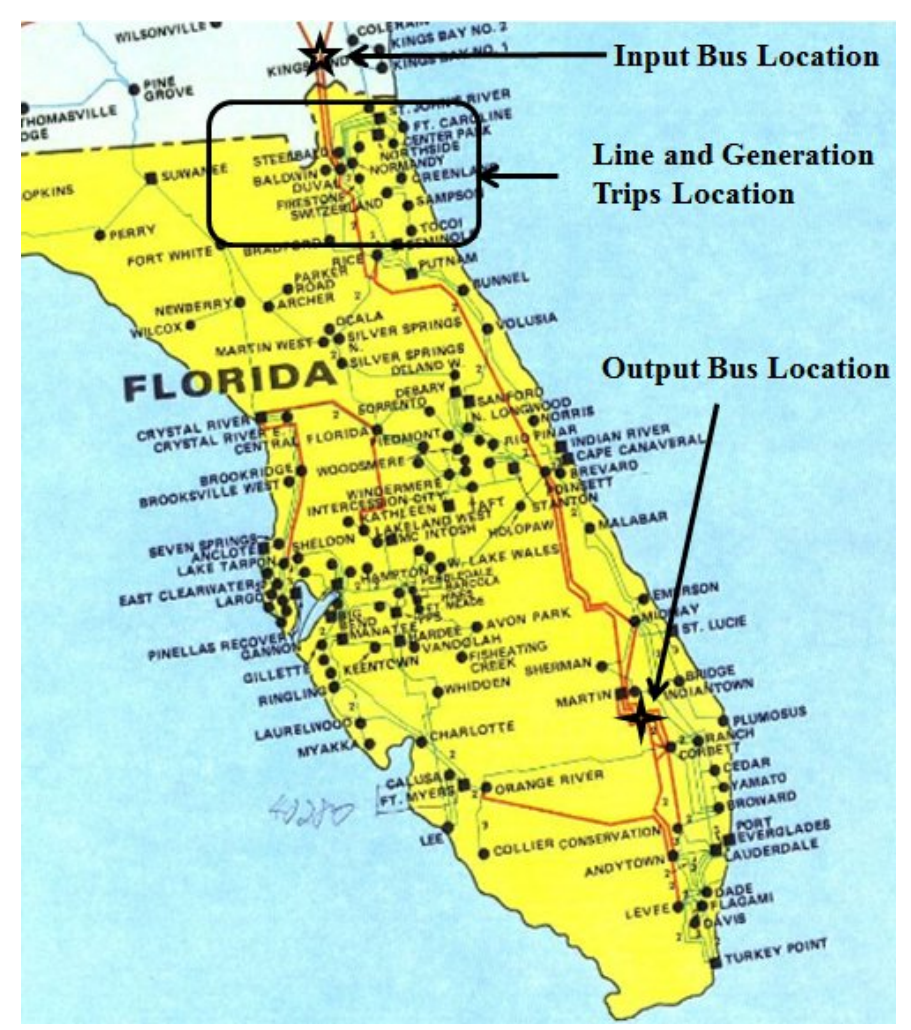

Fig.11. Map of Florida area power grid

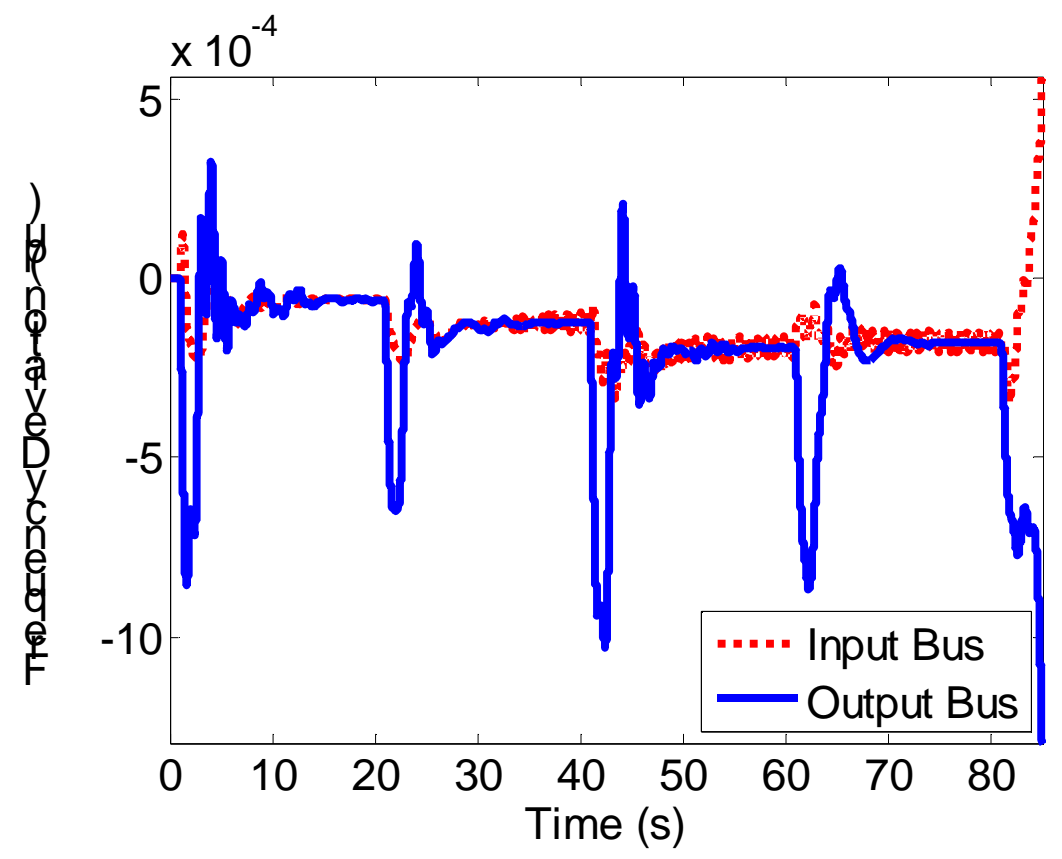

Fig.12. Frequency response following the disturbances 


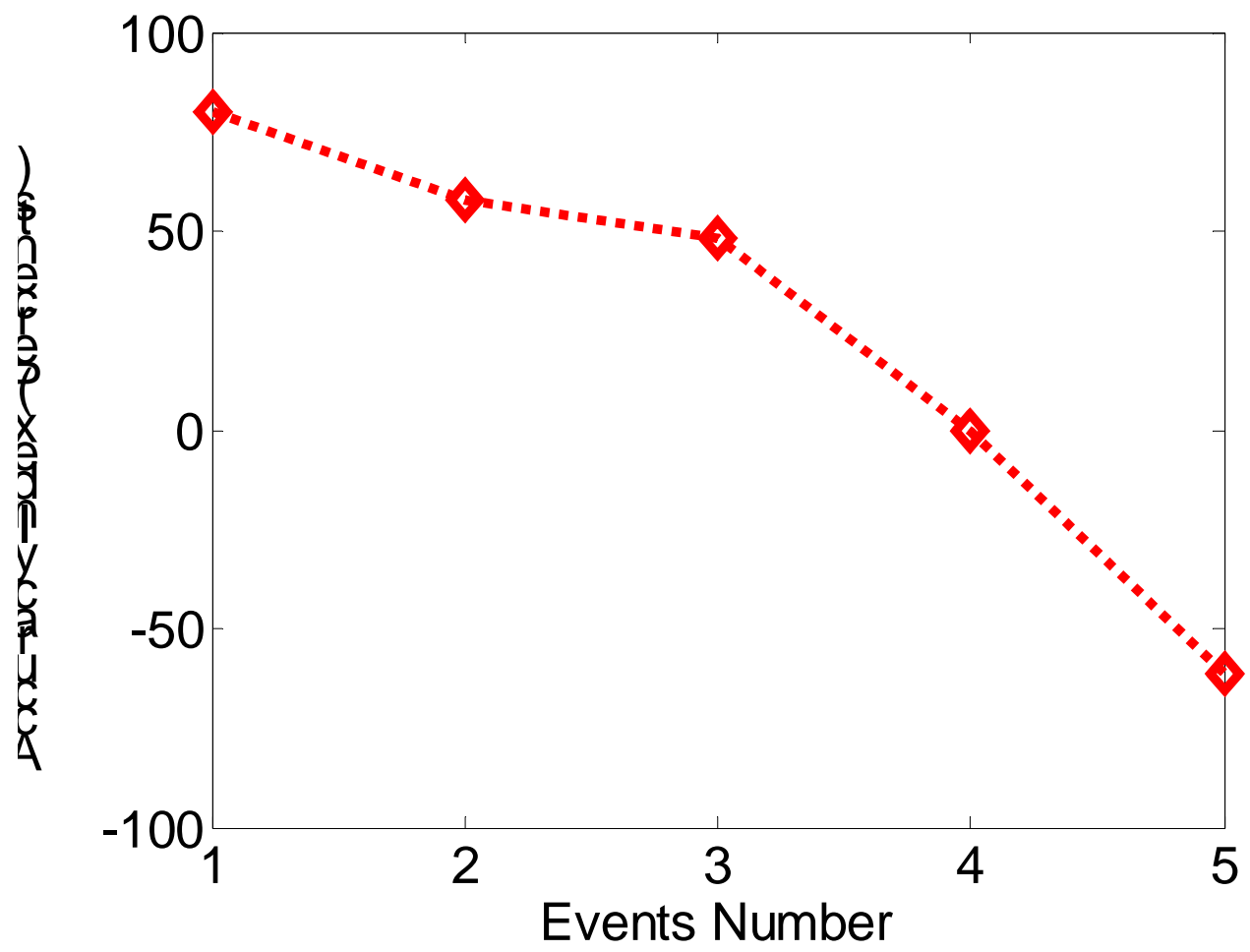

Fig.13. Accuracy indices of frequency response estimation following the sequence of disturbances 


\section{TABLE I}

BASIC INFORMATION OF THE 16,000-BUS EI DYNAMIC MODEL

\begin{tabular}{|c|c|}
\hline Total Bus Number & 16,013 \\
\hline Generator Number & 3248 \\
\hline $\begin{array}{c}\text { Total Operating } \\
\text { Generation Capacity }\end{array}$ & $590.7 \mathrm{GW}$ \\
\hline Total Load Capacity & $583.4 \mathrm{GW}$ \\
\hline
\end{tabular}

TABLE II

INFORMATION OF EXCITATION SOURCES AND ESTIMATED EVENT

\begin{tabular}{|c|c|c|}
\hline Location & Event Type & Amount (MW) \\
\hline \multirow{4}{*}{ New York } & Generation Trip & 839 \\
\cline { 2 - 3 } & Load Shedding & 809 \\
\cline { 2 - 3 } & Line Trip & 790 \\
\cline { 2 - 3 } & Line Fault & 800 \\
\hline Florida & Generation Trip & 810 \\
\hline
\end{tabular}

TABLE III

ACCURACY OF FREQUENCY ESTIMATION

\begin{tabular}{|c|c|}
\hline Excitation Sources & Frequency Estimation Accuracy Index \\
\hline Generation Trip & 92.2210 \\
\hline Load Shedding & 80.4704 \\
\hline Line Trip & 51.2044 \\
\hline Line Fault & 75.6219 \\
\hline
\end{tabular}

